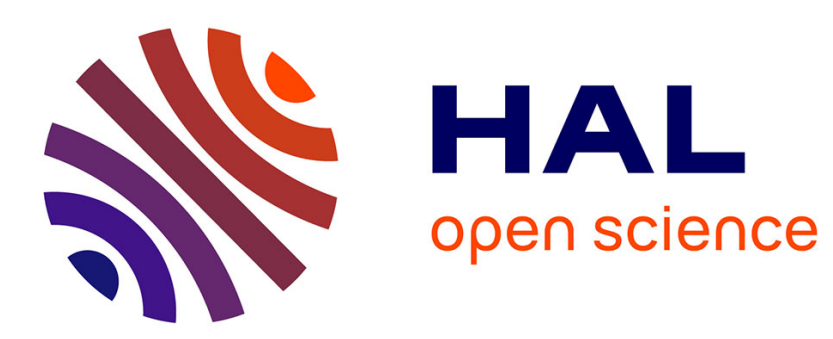

\title{
Étude de la résonance géante dipolaire dans la région de transition autour de $\mathrm{A}=190$
}

A. Veyssière, H. Beil, R. Bergère, P. Carlos, A. Leprêtre, A. de Miniac

\section{To cite this version:}

A. Veyssière, H. Beil, R. Bergère, P. Carlos, A. Leprêtre, et al.. Étude de la résonance géante dipolaire dans la région de transition autour de $\mathrm{A}=190$. Journal de Physique Lettres, 1975, 36 (11), pp.267-270. 10.1051/jphyslet:019750036011026700 . jpa-00231205

\section{HAL Id: jpa-00231205 https://hal.science/jpa-00231205}

Submitted on 1 Jan 1975

HAL is a multi-disciplinary open access archive for the deposit and dissemination of scientific research documents, whether they are published or not. The documents may come from teaching and research institutions in France or abroad, or from public or private research centers.
L'archive ouverte pluridisciplinaire HAL, est destinée au dépôt et à la diffusion de documents scientifiques de niveau recherche, publiés ou non, émanant des établissements d'enseignement et de recherche français ou étrangers, des laboratoires publics ou privés. 
Classification

Physics Abstracts

$4.340-4.470-4.480$

\title{
ÉTUDE DE LA RÉSONANCE GÉANTE DIPOLAIRE DANS LA RÉGION DE TRANSITION AUTOUR DE $A=190$
}

\author{
A. VEYSSIÈRE, H. BEIL, R. BERGÈRE, P. CARLOS, \\ A. LEPRETTRE et A. DE MINIAC
}

Département de Physique Nucléaire, C.E.N. Saclay, B.P. 2, 91190 Gif-sur-Yvette, France

(Reçu le 28 juillet 1975, accepté le 9 septembre 1975)

\begin{abstract}
Résumé. - La résonance géante des noyaux $\mathrm{W}, \mathrm{Re}, \mathrm{Ir}, \mathrm{Pt}$ et $\mathrm{Hg}$ a été étudiée avec des photons monochromatiques de 8 à $22 \mathrm{MeV}$, en mesurant les sections efficaces partielles $[\sigma(\gamma, \mathrm{n})+\sigma(\gamma, \mathrm{pn})]$ et $\sigma(\gamma, 2 \mathrm{n})$. Les formes observées pour ces résonances géantes correspondent bien à l'évolution prédite par le modèle collectif dynamique lorsqu'on passe d'un noyau à déformation permanente allongée $(\mathrm{W}, \mathrm{Re})$ à un noyau de forme aplatie ou triaxiale $\gamma$ instable $(\mathrm{Pt})$.
\end{abstract}

\begin{abstract}
Partial photoneutron cross-sections $[\sigma(\gamma, \mathrm{n})+\sigma(\gamma, \mathrm{pn})]$, and $\sigma(\gamma, 2 \mathrm{n})$ of $\mathbf{W}, \mathbf{R e}, \mathbf{I r}$, $\mathrm{Pt}$ and $\mathrm{Hg}$ were measured by means of monochromatic photons of $8 \mathrm{MeV} \leqslant E \leqslant 22 \mathrm{MeV}$ so as to study the giant resonance. The experimentally observed evolution of the shape of the GDR, as one proceeds from permanently deformed prolate nuclei ( $\mathrm{W}$ and $\mathrm{Re}$ ) towards oblate or even triaxial gamma unstable nuclei $(\mathrm{Pt})$, corresponds to the theoretical predictions of the dynamic collective model.
\end{abstract}

Pour les noyaux lourds compris entre les couches fermées en neutrons $N=82$ et $N=126$, les données expérimentales sur les spectres à basse énergie sont bien expliquées aujourd'hui par les surfaces d'énergie potentielle calculées $[1,2]$. On connaît deux zones de transition importantes dans cette région de masse [3] :

1) La zone autour de $N=88-90$ où les noyaux passent d'une forme quasi sphérique à une forme à déformation permanente allongée;

2) La zone autour de $A=190$ où les noyaux passent d'une déformation permanente allongée à une déformation qui, sans être franchement aplatie correspond à une instabilité pour les vibrations $\gamma$.

Or, pour tous les noyaux lourds, la distribution en énergie des états collectifs de la résonance géante (RGD) est bien prédite par le modèle collectif dynamique (DCM) qui prend en compte le couplage entre les oscillations de volume dipolaires et les vibrations à basse énergie de la surface nucléaire. Alors que les premiers modèles DCM ne pouvaient traiter que le cas des noyaux vibrateurs harmoniques ou rotateurs purs $[4,5]$, l'amélioration récente de ce modèle, fondée sur la description du noyau par sa surface d'énergie potentielle (PES) permet de considérer ce couplage même dans le cas des noyaux de transition [6]. On a ainsi obtenu un accord remarquable entre les prédic- tions de ce dernier modèle [7] et l'évolution observée dans notre laboratoire pour la forme de la RGD des isotopes de $\mathrm{Nd}$ et $\mathrm{Sm}$ (de $N=82$ à $N=92$ ) $[8,9]$.

Par contre, très peu de résultats expérimentaux existent pour la RGD des noyaux de $A$ voisin de 190. En particulier seules les RGD des noyaux ${ }^{186} \mathrm{~W}$ et ${ }^{197} \mathrm{Au}$ ont été étudiées avec des photons monochromatiques $[10,11]$ qui donnent accès directement à la forme de la section efficace sans passer par les méthodes de déconvolution hasardeuses qui sont nécessaires lorsqu'on utilise des spectres continus de photons de freinage. Cependant les surfaces d'énergie potentielle de ces noyaux sont maintenant suffisamment connues et ont récemment pu être utilisées [12] pour calculer la distribution des états dipolaires des isotopes d'osmium 184, 186, 188, 190 et 192. Cette distribution théorique montre une évolution depuis une structure dédoublée de la RGD caractéristique des noyaux à déformation allongée permanente $\left({ }^{184} \mathrm{Os},{ }^{186} \mathrm{Os}\right)$ jusqu'à une répartition plus étalée vers les hautes énergies pour le noyau triaxial ${ }^{192}$ Os qui est instable.

L'interprétation de la forme de la RGD de l'osmium naturel serait donc particulièrement compliquée. Par contre on peut remarquer une similitude importante entre les spectres rotationnels à basse énergie des couples ${ }^{184} \mathrm{~W}-{ }^{184} \mathrm{Os}$ et ${ }^{186} \mathrm{~W}-{ }^{186} \mathrm{Os}$, ainsi qu'entre les PES calculées correspondantes. De même, on peut 
remarquer la similitude des spectres de rotateur asymétrique $\gamma$ instable pour ${ }^{192} \mathrm{Os}$ et ${ }^{194} \mathrm{Pt}-{ }^{196} \mathrm{Pt}$, ce qui est bien reflété dans les surfaces d'énergie potentielle calculées Kumar et Baranger [13].

Il semble donc intéressant d'étudier systématiquement la RGD pour des noyaux couvrant cette zone de transition. Pour ces noyaux lourds, les voies de sortie sont essentiellement les voies neutroniques $(\gamma, \mathrm{n}), \sigma(\gamma, \mathrm{pn})$ et $(\gamma, 2 \mathrm{n})$. En utilisant le faisceau de photons monochromatiques d'énergie variable obtenu par annihilation en vol de positons [13] à l'accélérateur linéaire de Saclay AL 60 et un détecteur de neutrons [14] (scintillateur liquide de grande efficacité) $(\varepsilon \simeq 50 \%)$ nous avons mesuré séparément les sections efficaces $[\sigma(\gamma, \mathrm{n})+\sigma(\gamma, \mathrm{pn})]$ (Fig. 1) et $\sigma(\gamma, 2 \mathrm{n})$ (Fig. 2).

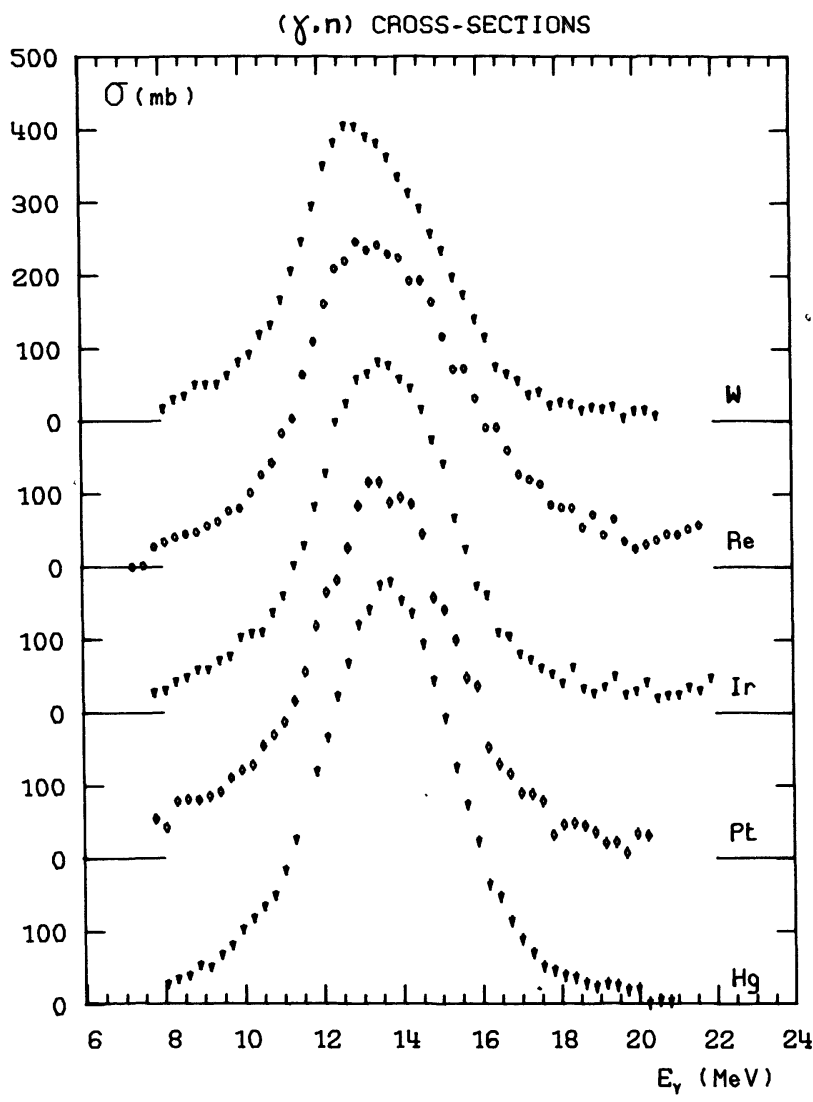

Fig. 1. - Sections efficaces partielles $\sigma(\gamma, \mathrm{n})+\sigma(\gamma, \mathrm{pn})$ des noyaux W, Re, Ir, Pt, Hg.

On peut ainsi reconstruire, à partir de ces deux voies de sortie principales, la section efficace d'absorption totale photonique dans la RGD (Fig. 3)

$$
\sigma_{\mathrm{T}}(E)=\sigma(\gamma, \mathrm{n})+\sigma(\gamma, \mathrm{pn})+\sigma(\gamma, 2 \mathrm{n})
$$

qui est la grandeur théoriquement calculée par le DCM.

On voit clairement que $\sigma_{\mathrm{T}}(E)$ pour $\mathrm{W}$, et $\mathrm{Re}$ montre le dédoublement de la RGD prévu par le modèle collectif pour les noyaux à déformation permanente allongée. En effet on peut représenter les sections

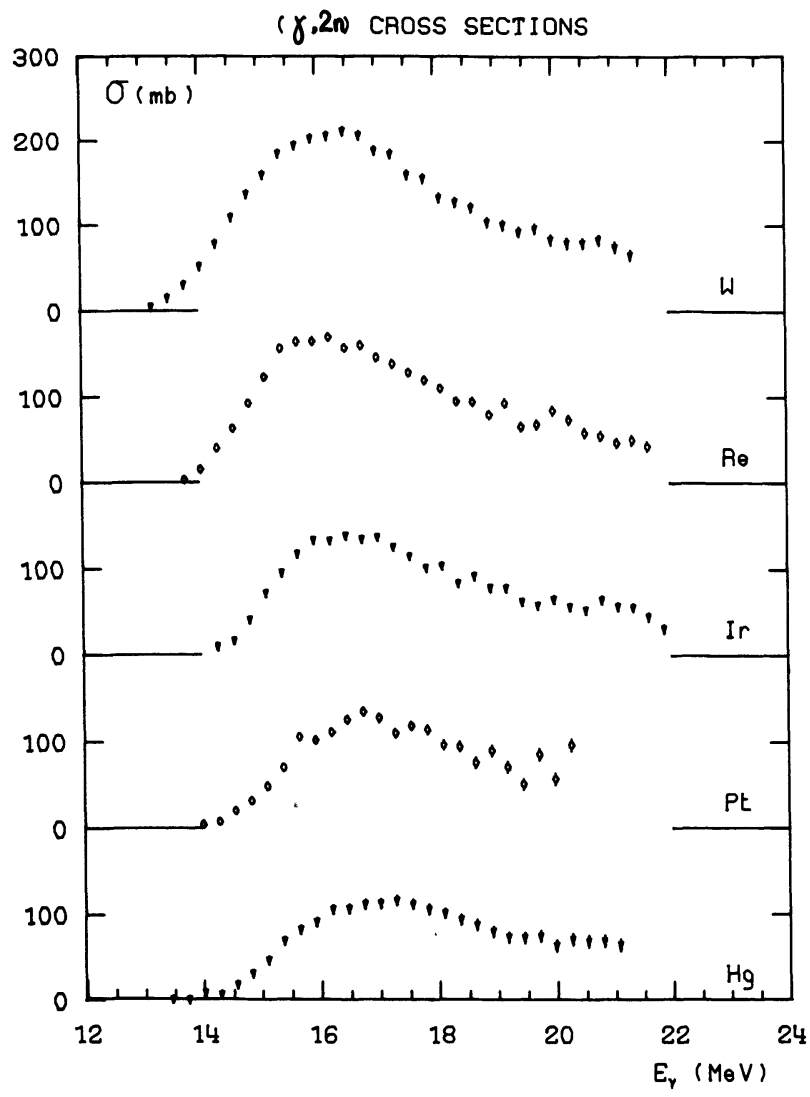

FIG. 2. - Sections efficaces partielles $\sigma(\gamma, 2 \mathrm{n})$ des noyaux W, $\mathrm{Re}, \mathrm{Ir}, \mathrm{Pt}, \mathrm{Hg}$.

TOTAL CROSS-SECTIONS

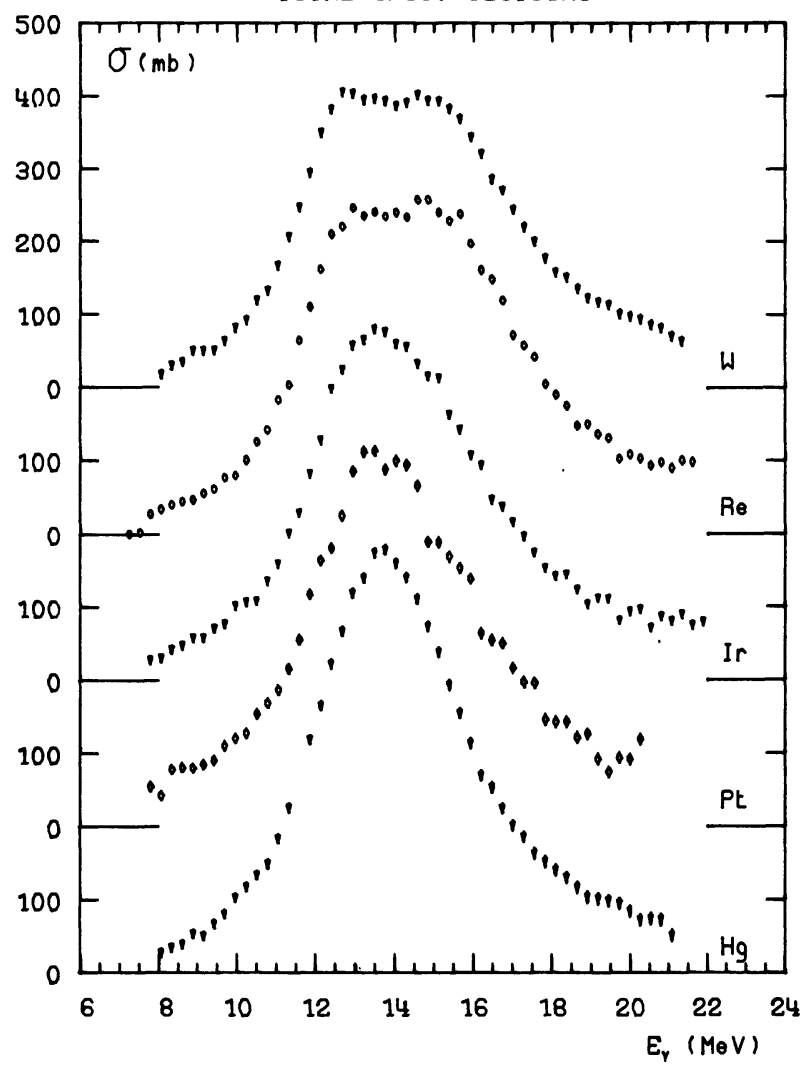

FIG. 3. - Sections efficaces totales $\sigma_{\mathrm{T}}(E)$ des noyaux $\mathrm{W}, \mathrm{Re}$, $\mathrm{Ir}, \mathrm{Pt}, \mathrm{Hg}$. 
efficaces photoneutroniques totales par deux raies de Lorentz suivant la relation :

$$
\begin{aligned}
\sigma_{\mathrm{L}}=\sigma_{1} \frac{\left(E \Gamma_{1}\right)^{2}}{\left(E^{2}-E_{1}^{2}\right)^{2}+\left(E \Gamma_{1}\right)^{2}}+ & \\
& \quad+\sigma_{2} \frac{\left(E \Gamma_{2}\right)^{2}}{\left(E^{2}-E_{2}^{2}\right)^{2}+\left(E \Gamma_{2}\right)^{2}}
\end{aligned}
$$

On obtient, pour $\mathrm{Re}$ et $\mathrm{W}$ un ensemble de paramètres $\sigma_{1}, \Gamma_{1}, E_{1}$ et $\sigma_{2} E_{2} \Gamma_{2}$ donnés dans le tableau I.

\begin{tabular}{ccccccc}
\multicolumn{7}{c}{ TABLEAU I } \\
& $\sigma_{1}$ & $\Gamma_{1}$ & $E_{1}$ & $\sigma_{2}$ & $\Gamma_{2}$ & $E_{2}$ \\
& $\mathrm{mb}$ & $\mathrm{MeV}$ & $\mathrm{MeV}$ & $\mathrm{mb}$ & $\mathrm{MeV}$ & $\mathrm{MeV}$ \\
$\mathrm{W}$ & - & - & - & - & - & - \\
$\mathrm{Re}$ & 300 & 3,2 & 12,7 & 290 & 4,3 & 15,4 \\
& 310 & 3,3 & 12,8 & 330 & 4,45 & 15,4
\end{tabular}

Bien que le rapport des surfaces des deux raies

$$
\frac{\sigma_{2} \gamma_{2}}{\sigma_{1} \gamma_{1}}=1,4
$$

et non pas 2 comme dans le modèle statique simple, on peut néanmoins calculer le moment quadrupolaire intrinsèque moyen de ces deux noyaux par la formule [15]

$$
Q_{0}=\frac{2}{5} R_{0}^{2} Z A^{2 / 3} \frac{d^{2}-1}{d^{2 / 3}}
$$

avec $E_{2} / E_{1}=0,911 d+0,089$. Si on prend $R_{0}=1,2 \mathrm{fm}$ on trouve, pour $\mathrm{W}$ et $\mathrm{Re}, Q_{0}=6 \pm 0,5$ barns en bon accord avec les valeurs recommandées par Löbner et al. [16] qui varient de $6,4\left({ }^{182} \mathrm{~W}\right)$ à $5,9\left({ }^{186} \mathrm{~W}\right)$ et de 5,99 barns $\left({ }^{185} \mathrm{Re}\right)$ à $5,75\left({ }^{187} \mathrm{Re}\right)$. Ce dédoublement en deux raies est bien représenté par le spectre théorique des états dipolaires calculés par R. Sedlmayr et al. [12] pour ${ }^{184}$ Os (Fig. 4a).

Quand $A$ croît, on observe une disparition de ce dédoublement de la RGD et une diminution de la largeur à mi-hauteur : $\Gamma=5,2 \mathrm{MeV}, 5 \mathrm{MeV}$ et $4,4 \mathrm{MeV}$ respectivement pour $\mathrm{Ir}, \mathrm{Pt}$ et $\mathrm{Hg}$ on observe surtout une dissymétrie marquée de la RGD par rapport à l'énergie $E_{0}$ correspondant au maximum de la section efficace. Cette dissymétrie n'était pas observée pour la RGD des noyaux sphériques à simple couche fermée $[8,9](N=82)$ ou à double couche fermée $\left({ }^{208} \mathrm{~Pb}\right)$ [11]. Cet étalemènt de la RGD vers des énergies supérieures à $E_{0}$ est bien représenté par les prédictions théoriques [12] du DCM pour
${ }^{192}$ Os où plusieurs raies à haute énergie caractérisent l'instabilité du noyau vis-à-vis des vibrations $\gamma$ (Fig. 4b). On sait, par ailleurs, que lorsqu'on atteint ${ }^{208} \mathrm{~Pb}$, noyau sphérique rigide, la RGD se rétrécit encore $(\Gamma=4 \mathrm{MeV})$ et devient beaucoup plus symétrique [11]. Cette évolution est déjà sensible quand on passe de $\mathrm{Ir}$ à $\mathrm{Hg}$.

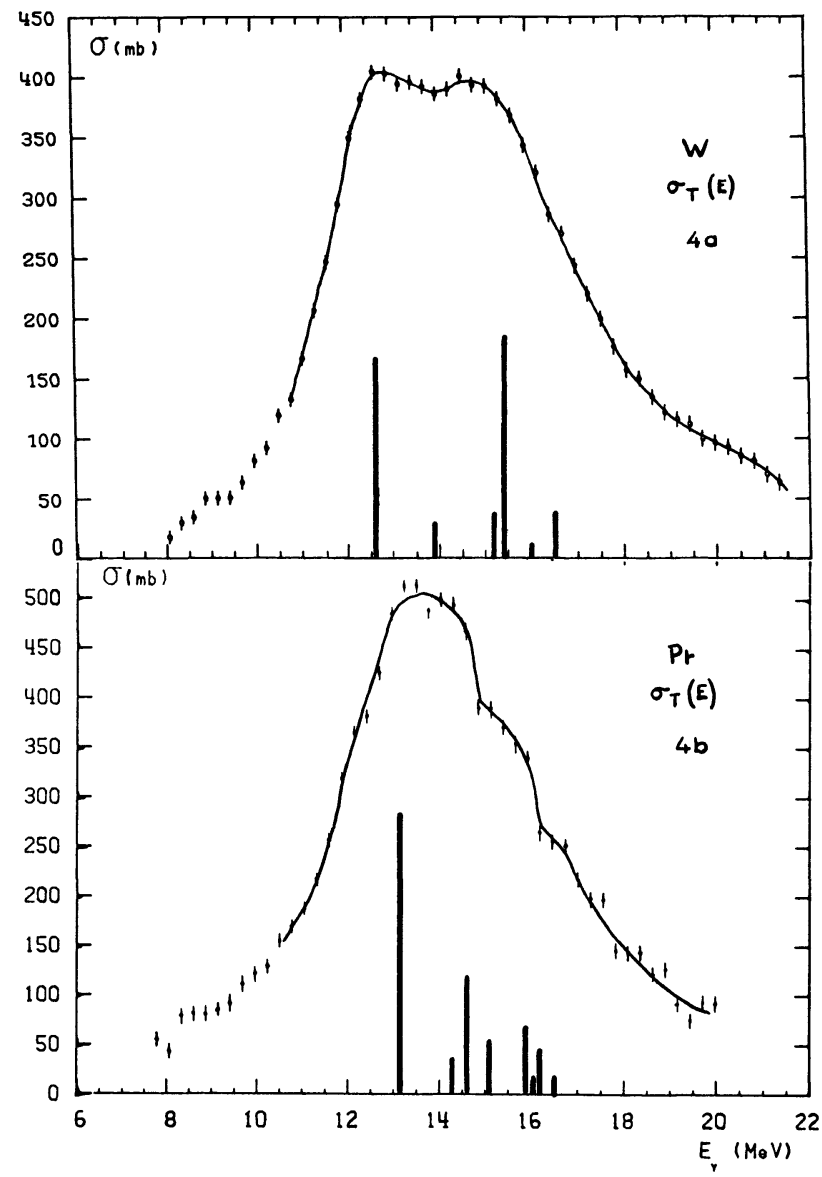

Fig. 4. - Comparaison des sections efficaces totales expérimentales $\sigma_{\mathrm{T}}(E)$ des noyaux $\mathrm{W}$ et $\mathrm{Pt}$ avec les prédictions du modèle collectif dynamique [12] positionnées de façon à faire coïncider les énergies moyennes observées et calculées de la RGD.

On peut enfin noter que le DCM prévoit la position en énergie des principaux états dipolaires mais ne prévoit pas la largeur d'amortissement $\Gamma^{\downarrow}$ qui doit être associée à chacun de ces états. Une comparaison systématique aux résultats expérimentaux [18] ainsi qu'une étude théorique récente [17] montrent qu'il faut considérer les valeurs $\Gamma^{\downarrow}$ voisines de 3 à $4 \mathrm{MeV}$. Ceci rend sans espoir une identification individuelle, par absorption de photons, des états dipolaires prévus par le DCM pour un noyau de forme triaxiale ou oblate $\gamma$ instable.

\section{Bibliographie}

[1] Kumar, K., Baranger, H., Nucl. Phys. A 122 (1968) 273.

[2] Mosel, U., Greiner, W., Z. Phys. 217 (1968) 256.

[3] Colloque sur les noyaux de transition. Orsay 1971.

Cline, D. (p. 4), Kumar, K. (p. 35), Gneuss, G., Bernus, L. V., SHNEIDER, V., Greiner, W. (p. 53).

[4] Arenhövel, H., Danos, M., Greiner, W., Phys. Rev. 157
(1967) 1109.

[5] Huber, M. G., Danos, M., Weber, H. J., Greiner, W., Phys. Rev. 155 (1967) 1073.

[6] Rezwani, V., Gneuss, G., Arenhövel, H., Phys. Rev. Lett. 25 (1970) 1667.

[7] Rezvani, V., Brandt, M., Sedlmayr, R., Bernus, L., SchneI- 
DER, U., GReINER, W., Proc. Int. Conf. on nuclear structure studies Sendai, Japan (1972).

[8] Carlos, P., Beil, H., Bergère, R., Leprêtre, A., Veyssière, A., Nucl. Phys. A 172 (1971) 437.

[9] Carlos, P., Beil, H., Bergère, R., Leprêtre, A., de Miniac, A., Veyssière, A., Nucl. Phys. A 225 (1974) 171.

[10] Berman, M. L., Kelly, M. A., Bramblett, R. L., Caldwell, J. T., Davis, H. S., Fultz, S. C., Phys. Rev. 185 (1969) 1576.

[11] Veyssière, A., BeIl, H., Bergère, R., Carlos, P., Leprêtre, A., A 159 (1970) 561.

[12] Sedlmayr, R., Sedlmayr, M., Greiner, W., Nucl. A 232 (1974) 465 .
[13] Audit, G., Beio, H., Bergère, R., De Botton, N., TAmas, G., VeYssière, A., Nucl. Instrum. 79 (1970) 203.

[14] BeIL, H., Bergère, R., VeYsSIÈre, A., Nucl. Instrum. 67 (1969) 293.

[15] Danos, M., Nucl. Phys. 5 (1958) 23.

[16] LöBnER, K. E. G., VetTer, N., HöNIG, V., Nucl. Data Tables $7 / 5$ (1970) 495.

[17] Mshelia, E. D., Roos, K., Greiner, W., Nucl. Phys. A 212 (1973) 157.

[18] Carlos, P., Bergère, R., Beil, H., Leprêtre, A., Veyssière, A., Nucl. Phys. A 219 (1974) 61. 